Mathematical Research Letters 5, 185-190 (1998)

\title{
THE EXTENDED FUTURE TUBE IS A DOMAIN OF HOLOMORPHY
}

\author{
XIANG-Yu ZHOU
}

\section{Introduction}

In this note, we present an approach to proving the extended future tube conjecture, which asserts that the extended future tube is a domain of holomorphy. Our main result is that the extended future tube conjecture is valid. Our basic tools for the proof are the minimum principle due to C.O. Kiselman and J. J. Loeb and the slice theory due to D. Luna. Our proof is based on several simple key observations. The starting observation is to relate the conjecture to the minimum principle. After applying the minimum principle and the slice theory, we reduce our proof to several more or less elementary (though less obvious) propositions (cf. §3). The details of the proof will appear elsewhere [18]. In this note, we present our main idea and main technical propositions of the proof.

\section{The extended future tube conjecture: Background and reformulation}

1.1. The extended future tube conjecture originated naturally from the axiomatic quantum field theory nearly four decades ago when properties of the Wightman functions were studied. Some mathematicians and physicists including N. N. Bogoliubov, K. Hepp, R. Jost, V. S. Vladimirov, and A. S. Wightman posed or tackled this conjecture (cf. R. Jost [2], R. F. Streater and A. S. Wightman [11], V. S. Vladimirov [12, 13], V. S. Vladimirov-V. V. Zharinov [14], A. G. Sergeev-V. S. Vladimirov [7], A. G. Sergeev-X. Y. Zhou [8], A. G. Sergeev-P. Heinzner [9], B. I. Zav'yalov-V. B. Trushin [16], and references therein). This conjecture has consequences in physics.

Definition 1. Let $M^{4}$ be the Minkowski space with the Lorentz metric: $x \cdot y=$ $x_{0} y_{0}-x_{1} y_{1}-x_{2} y_{2}-x_{3} y_{3}$, where $x=\left(x_{0}, x_{1}, x_{2}, x_{3}\right), y=\left(y_{0}, y_{1}, y_{2}, y_{3}\right) \in R^{4}$. The future (past) light cone $V^{+}\left(V^{-}\right)$is defined as

$$
V^{+}:=\left\{y \in M^{4}: y^{2}>0, y_{0}>0\right\}, \quad\left(V^{-}=-V^{+}\right) .
$$

The future and past tubes $\tau^{ \pm}$are defined as the correspording tube domains $\tau^{ \pm}=R^{4}+i V^{ \pm}$in $\mathbb{C}^{4}$. The $N$-point future (past) tube is defined as $\tau_{N}^{+}=$ $\tau^{+} \times \cdots \times \tau^{+} N$-times $\left(\tau_{N}^{-}=\tau^{-} \times \cdots \times \tau^{-} N\right.$-times $)$.

Received June 24, 1997.

This work was partially supported by the Chinese NSF. 
Definition 2. The identity component of the Lorentz group is called the restricted Lorentz group $L_{+}^{\uparrow}=S O_{+}(1,3)$. The identity component of the complex Lorentz group is called the proper complex Lorentz group $L_{+}(\mathbb{C})=S O(1,3, \mathbb{C}) \stackrel{\simeq}{\cong}$ $S O(4, \mathbb{C})$.

The group $L_{+}^{\uparrow}$ is a connected closed real form of $L_{+}(\mathbb{C})$. The sets $V^{+}$and $\tau^{+}$ are $L_{+}^{\uparrow}$-invariant with respect to the usual linear action.

Definition 3. Let $L_{+}(\mathbb{C})$ act diagonally on $\mathbb{C}^{4 N}$, i.e., for $z=\left(z^{(1)}, \cdots, z^{(N)}\right) \in$ $\mathbb{C}^{4 N}, \Lambda z=\left(\Lambda z^{(1)}, \cdots, \Lambda z^{(N)}\right)$, where $\Lambda \in L_{+}(\mathbb{C}), z^{(1)}, \cdots, z^{(N)} \in \mathbb{C}^{4}$. The extended ( $N$-point) future tube is defined as $\tau_{N}^{\prime}=L_{+}(\mathbb{C}) \cdot \tau_{N}^{+}=\{\Lambda z: z \in$ $\left.\tau_{N}^{+}, \Lambda \in L_{+}(\mathbb{C})\right\}$ which is obviously a domain in $\mathbb{C}^{4 N}$.

The extended future tube conjecture asserts that $\tau_{N}^{\prime}$ is a domain of holomorphy for $N \geq 3$. It is known that $\tau_{1}^{\prime}$ and $\tau_{2}^{\prime}$ are domains of holomorphy (cf. Vladimirov [12]).

1.2. The extended future tube conjecture is regarded to be a very natural conjecture from the physical point of view. From a mathematical point of view, it is also quite natural in view of the following BHW Theorem and Streater's Theorem.

Theorem [Bargman, Hall, and Wightman (BHW)]. An $L_{+}^{\uparrow}$-invariant holomorphic function on $\tau_{N}^{+}$can be extended to an $L_{+}(\mathbb{C})$-invariant holomorphic function on $\tau_{N}^{\prime}(c f .[2,11])$.

A natural question arises, i.e., can these holomorphic functions be extended further? This is equivalent to asking if $\tau_{N}^{\prime}$ is a domain of holomorphy.

Theorem (Streater). A holomorphic function on the Dyson domain given by $\tau_{N}^{+} \cup \tau_{N}^{-} \cup J$ (where $J:=\tau_{N}^{\prime} \cap M^{4 N}$ is the set of Jost points which was proved to exist and characterized by $R$. Jost) can be extended to a holomorphic function on $\tau_{N}^{\prime}(c f .[2,11])$.

A natural question is to construct the envelope of holomorphy of the Dyson domain $\tau_{N}^{+} \cup \tau_{N}^{-} \cup J$. (This question is mentioned in the article "Quantum field theory" of the Russian great dictionary "Encyclopaedia of Mathematics".) The extended future tube conjecture means that this envelope of holomorphy is exactly the extended future tube $\tau_{N}^{\prime}$.

1.3. The Pauli mapping is defined as follows:

$$
\begin{gathered}
P: \mathbb{C}^{4} \longrightarrow \mathbb{C}[2 \times 2] \\
z=\left(z_{0}, z_{1}, z_{2}, z_{3}\right) \mapsto Z:=\left(\begin{array}{cc}
z_{0}+z_{3} & z_{1}-i z_{2} \\
z_{1}+i z_{2} & z_{0}-z_{3}
\end{array}\right) .
\end{gathered}
$$


Here, $\mathbb{C}[2 \times 2]$ consists of all $2 \times 2$ complex matrices. Let $H$ be the image of $\tau^{+}$ under $P$. One can easily check that

$$
H=\left\{Z \in \mathbb{C}[2 \times 2]: \frac{Z-\bar{Z}^{\prime}}{2 i}>0\right\}
$$

where $\bar{Z}^{\prime}$ is the conjugate transpose of $Z$.

For every $A \in S L(2, \mathbb{C})$, the linear transformation of $\mathbb{C}[2 \times 2]: Z \mapsto A Z \bar{A}^{\prime}$ determines under the Pauli mapping a linear transformation of $\mathbb{C}^{4}: z \mapsto g_{A} \cdot z$, where $g_{A} \in L_{+}^{\uparrow}$. This determines a universal double covering homomorphism $\rho$ : $S L(2, \mathbb{C}) \rightarrow L_{+}^{\uparrow}$. Similarly, the linear transformation of $\mathbb{C}[2 \times 2]: Z \mapsto A Z B^{-1}$, $A, B \in S L(2, \mathbb{C})$, determines under the Pauli mapping a linear transformation of $\mathbb{C}^{4}: z \mapsto g_{(A, B)} \cdot z$, where $g_{(A, B)} \in L_{+}(\mathbb{C})$. This determines a universal double covering homomorphism $\tilde{\rho}: S L(2, \mathbb{C}) \times S L(2, \mathbb{C}) \rightarrow L_{+}(\mathbb{C})$.

Consider an action of $S L(2, \mathbb{C}) \times S L(2, \mathbb{C})$ on $\mathbb{C}^{N}[2 \times 2]$ given by

$$
(A, B) \cdot Z=\left(A Z_{1} B^{-1}, \cdots, A Z_{N} B^{-1}\right),
$$

where

$$
A, B \in S L(2, \mathbb{C}), \quad Z_{1}, \cdots, Z_{N} \in \mathbb{C}[2 \times 2] .
$$

The induced real action of $S L(2, \mathbb{C})$ is given by

$$
A \cdot Z=\left(A,\left(\bar{A}^{\prime}\right)^{-1}\right) \cdot Z=\left(A Z_{1} \bar{A}^{\prime}, \cdots, A Z_{N} \bar{A}^{\prime}\right),
$$

where $A \in S L(2, \mathbb{C})$.

Set $\widetilde{P}:=P \times \cdots \times P: \mathbb{C}^{4 N} \rightarrow \mathbb{C}^{N}[2 \times 2]$. Denote by

$$
\begin{aligned}
H_{N}^{\prime} & =\widetilde{P}\left(\tau_{N}^{\prime}\right)=(S L(2, \mathbb{C}) \times S L(2, \mathbb{C})) \cdot H_{N} \\
& =\left\{\left(A Z_{1} B^{-1}, \cdots, A Z_{N} B^{-1}\right):\left(Z_{1}, \cdots, Z_{N}\right) \in H_{N}, A, B \in S L(2, \mathbb{C})\right\},
\end{aligned}
$$

where $H_{N}=H \times \cdots \times H, N$-times. Note that $H_{N}=\widetilde{P}\left(\tau_{N}^{+}\right)$is $S L(2, \mathbb{C})$ invariant, and $\widetilde{P}^{-1}$ is $\tilde{\rho}$-equivariant and biholomorphic. Therefore, the extended future tube conjecture is equivalent to the assertion that $H_{N}^{\prime}$ is a domain of holomorphy for $N \geq 3$.

\section{Preparatory tools}

2.1. Let $X$ be a complex manifold, $G_{\mathbb{C}}$ a connected complex Lie group, and $G_{\mathbb{R}}$ a connected closed real form of $G_{\mathbb{C}}$. Denote $\psi: G_{\mathbb{C}} \rightarrow G_{\mathbb{C}} / G_{\mathbb{R}}$, and $p: X \times G_{\mathbb{C}} \rightarrow X$ the natural projections. The group $G_{\mathbb{C}}$ acts on $X \times G_{\mathbb{C}}$ on the right by:

$$
\begin{aligned}
& \left(X \times G_{\mathbb{C}}\right) \times G_{\mathbb{C}} \longrightarrow X \times G_{\mathbb{C}} \\
& ((x, g), h) \longmapsto(x, g h) .
\end{aligned}
$$


Let $\Omega \subset X \times G_{\mathbb{C}}$ be a right $G_{\mathbb{R}}$-invariant domain and have connected fibres of $p$, and $u \in C^{\infty}(\Omega)$ be a right $G_{\mathbb{R}}$-invariant function. The function $u$ naturally induces a smooth function $\dot{u}(x, \psi(g))$ on $\dot{\Omega}:=\left(i d_{X}, \psi\right)(\Omega)$. Suppose that

(1) $u$ is p.s.h on $\Omega$,

(2) $\forall x \in p(\Omega), u(x, \cdot)$ is strictly p.s.h. on $\Omega_{x}=\Omega \cap p^{-1}(x)$, and

(3) $\dot{u}(x, \cdot)$ is exhaustive on $\dot{\Omega}_{x}=\psi\left(\Omega_{x}\right)$,

then the minimum principle asserts that $v(x)=\inf _{g \in \Omega_{x}} u(x, g)$ is $C^{\infty}$ and p.s.h. on $p(\Omega)$.

Remark 1. C. O. Kiselman in [3] first obtained the minimum principle when $X=\mathbb{C}^{n}, G_{\mathbb{C}}=\mathbb{C}^{m}, G_{\mathbb{R}}=\operatorname{Im} \mathbb{C}^{m}$. J. J. Loeb in [4] generalized Kiselman's result to the present general case.

Remark 2. One can seemingly weaken condition (3), replacing it by the condition that $\dot{u}(x, \cdot)$ attains its minimum on $\dot{\Omega}_{x}$. Our first proof used this seemingly weaker version of the minimum principle, so that the proof is more complicated. However, the essential point of our proof shows that one can actually use the above version of the minimum principle, so that the proof can be considerably simplified.

2.2. In this section, let $G_{\mathbb{C}}$ be a connected complex reductive Lie group, $X$ be

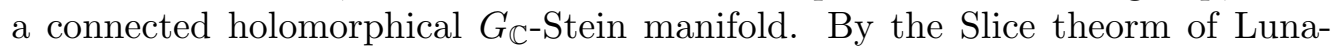
Snow (cf. Luna [5], Snow [9]), the categorical quotient $X / / G_{\mathbb{C}}$ is a normal Stein space and $\pi: X \rightarrow X / / G_{\mathbb{C}}$ is holomorphic (for the definition of the categorical quotient, cf. Snow [9]). It is known that $\pi$ is open, semi-proper, surjective, submersive (i.e. $X / / G_{\mathbb{C}}$ carries the quotient topology), and each fibre is connected and contains a unique closed orbit (cf. Snow [9]).

One can naturally introduce a partial order on the set of all closed $G_{\mathbb{C}}$ orbits, i.e., for two closed $G_{\mathbb{C} \text {-orbits }} G_{\mathbb{C}} \cdot x$ and $G_{\mathbb{C}} \cdot y$, we say $G_{\mathbb{C}} \cdot x>G_{\mathbb{C}} \cdot y$ if and only if there exists an equivariant holomorphic mapping from $G_{\mathbb{C}} \cdot x$ onto $G_{\mathbb{C}} \cdot y$. The maximal element must exist and is called the principal orbit; the corresponding isotropy group at the principal orbit is called the principal isotropy group. By the Slice theorem of Luna-Snow, the principal isotropy groups are conjugate, and a closed orbit $G_{\mathbb{C}} \cdot x$ is principal if and only if $\pi(x)$ is a regular point of $X / / G_{\mathbb{C}}$ and $\pi: X \rightarrow X / / G_{\mathbb{C}}$ is nondegenerate at $x$.

Denote $X^{\prime}:=\left\{x \in X: G_{\mathbb{C}} \cdot x\right.$ is closed and principal $\}, X_{p r}:=\pi^{-1}\left(\pi\left(X^{\prime}\right)\right)$, $S:=\left\{x \in X: \operatorname{rank}_{x} \pi<\operatorname{dim}\left(X / / G_{\mathbb{C}}\right)\right\}$, and $\operatorname{Sing}\left(X / / G_{\mathbb{C}}\right)$ the singular locus of $X / G_{\mathbb{C}}$. Then $\left(X_{p r}\right)^{c}=\pi^{-1}(\pi(S)) \cup \pi^{-1}\left(\operatorname{Sing}\left(X / G_{\mathbb{C}}\right)\right.$ ). Since $S$ is a $G_{\mathbb{C}^{-i n v a r i a n t}}$ analytic set in $X$, by semi properness of $\pi$, then $\pi(S)$ is also an analytic set in $X / / G_{\mathbb{C}}$. Therefore $X_{p r}$ is Zariski open in $X$. The set $\pi\left(X^{\prime}\right)$ is the so called principal model or principal stratum.

If $X$ has FPIG (i.e., all principal isotropy groups are finite), then $X^{\prime}=X_{p r}$, and $\pi^{-1}(\pi(x))=G_{\mathbb{C}} \cdot x$ for $x \in X^{\prime}$. 


\section{Proof of the reformulation of the extended future tube conjecture}

Our starting point is a simple and less obvious observation which is to relate the conjecture to the minimum principle which is stated in $\S 2.1$. In order to use the minimum principle, we make the following two simple observations.

Proposition 1. The Bergman kernel of $H_{N}$ is $S L(2, \mathbb{C})$-invariant strictly p.s.h.; in fact, $K_{H_{N}}(Z, \bar{Z})=c \prod_{j=1}^{N} 1 / \operatorname{det}\left(\operatorname{Im} Z_{j}\right)^{4}$, where $Z=\left(Z_{1}, \cdots, Z_{N}\right)$, c is a positive constant, $\operatorname{Im} Z_{j}=\left(Z_{j}-\bar{Z}_{j}^{\prime}\right) / 2 i$.

Proposition 2. The space $\mathbb{C}^{N}[2 \times 2]$ has FPIG with respect to the $S L(2, \mathbb{C}) \times$ $S L(2, \mathbb{C})$ action for $N \geq 3$. Denote by

$A=\mathbb{C}^{N}[2 \times 2] \backslash\left\{Z \in \mathbb{C}^{N}[2 \times 2]:(S L(2, \mathbb{C}) \times S L(2, \mathbb{C})) \cdot Z\right.$ is closed and principal $\}$.

Hence $A$ is an $S L(2, \mathbb{C}) \times S L(2, \mathbb{C})$-invariant analytic (actually, algebraic) set in $\mathbb{C}^{N}[2 \times 2]$ by Luna's slice theory (cf.2.2.).

The idea of the proof of the conjecture is to construct a p.s.h. exhaustion function on $H_{N}^{\prime}$ from a special strictly p.s.h. function on $H_{N}$. Choose a special $S L\left(2, \mathbb{C}\right.$ )-invariant s.p.s.h. function $\varphi$ on $H_{N}$ (for example, choose $\varphi$ as $K_{H_{N}}(Z, \bar{Z})$ or $\sum_{j=1}^{N} 1 / \operatorname{det}\left(\operatorname{Im} Z_{j}\right)^{4}$, or $\left.\sum_{j=1}^{N} 1 / \operatorname{det}\left(\operatorname{Im} Z_{j}\right)\right)$. Consider the holomorphic mapping

$$
\begin{aligned}
& \rho: \mathbb{C}^{N}[2 \times 2] \times(S L(2, \mathbb{C}) \times S L(2, \mathbb{C})) \longrightarrow \mathbb{C}^{N}[2 \times 2] \\
& (Z,(A, B)) \longmapsto A^{-1} Z B .
\end{aligned}
$$

The set $\Omega:=\rho^{-1}\left(H_{N}\right) \subset \mathbb{C}^{N}[2 \times 2] \times(S L(2, \mathbb{C}) \times S L(2, \mathbb{C}))$ has connected fibres of $p$ since $H_{N}$ is orbit connected. (This fact is an essential part of the BHW Theorem. For a definition of orbit connectedness, cf. Zhou [17]). The smooth function $\varphi\left(A^{-1} Z B\right)$ is defined and p.s.h on $\Omega$.

Denote $v(Z):=\inf _{(A, B) \in \Omega_{Z}} \varphi\left(A^{-1} Z B\right)$. Then $v(Z)$ is upper semicontinuous, and actually continuous, on $p(\Omega)=H_{N}^{\prime}$. Consider $\Omega^{\prime}:=\rho^{-1}\left(H_{N} \backslash A\right)$ which still has connected fibres of $p$ since $H_{N} \backslash A$ is still orbit connected. The function $\varphi\left(A^{-1} Z B\right)$ is strictly p.s.h. on $\left(\Omega^{\prime}\right)_{Z}=\Omega_{Z}$ for any fixed $Z \in p\left(\Omega^{\prime}\right)=H_{N}^{\prime} \backslash A$.

In order to use the minimum principle finally, we still need the following.

Proposition 3. The function $\dot{\varphi}$ induced by $\varphi\left(A^{-1} Z B\right)$ on $\left(\Omega^{\prime}\right)_{Z}$ is an exhaustion function on $\psi\left(\left(\Omega^{\prime}\right)_{Z}\right)$ for $Z \in p\left(\Omega^{\prime}\right)$.

Now using the minimum principle (Propositions 1, 2, 3 make it possible), one has that $v(Z)$ is p.s.h. on $H_{N}^{\prime} \backslash A$. By the extension theorem for p.s.h functions (cf. Gunning [1]), $v(Z)$ is p.s.h. in $H_{N}^{\prime}$. Our final step to prove the conjecture is the following. 
Proposition 4. The function $v(Z)$ goes to infinity as $Z$ goes to a boundary point of $H_{N}^{\prime}$.

Although the proofs of Proposition 3 and Proposition 4 are less obvious, they are more or less elementary.

\section{Acknowledgements}

The author would like to express his best regards to his teacher Prof. Lu Qikeng on the occasion of his seventieth birthday. He would also like to thank Prof. Wang Shikun for his help in typing this note and Prof. Dai Xingshen for his help in improving the English in the note. He is also grateful to Professors Ding Weiyue, Liu Kefeng, Tian Gang, Wang Youde, Wu Siye, and Zhang Weiping for their encouragements and interests in the present work.

\section{References}

1. R. C. Gunning, Introduction to holomorphic functions of several variables, Wadsworth Brooks/Cole Math. Ser., vol. 1-3, Wadsworth \& Brooks/Cole Advanced Books \& Software, Monterey, CA, 1990.

2. R. Jost, The general theory of quantized fields, Amer. Math. Soc., Providence, R.I., 1965.

3. C. O. Kiselman, The partial Legendre transformation for plurisubharmonic functions, Invent. Math. 49 (1978), 137-148.

4. J. J. Loeb, Actions d'une forme de Lie réelle d'un groupe de Lie conmplexe sur les fonctions plurisousharmoniques, Ann. Inst. Fourier 35 (1985), 59-97.

5. D. Luna, Slice étales, Bull. Soc. Math. France. Mem. 33 (1973), 81-105.

6. D. Mumford, J. Forgaty, and F. Kirwan, Geometric invariant theory, (3rd edition), Ergeb. Math. Grenzgeb. (2), 34, Springer-Verlag, 1994.

7. A. G. Sergeev and V. S. Vladimirov, Complex analysis in the future tube, Encyclopaedia of Math. Sci., Vol. 8 (Several Complex Variables, II), Springer-Verlag, 1994.

8. A. G. Sergeev and Xiang-Yu Zhou, On invariant domains of holomorphy, (in Russian), Proc. Steklov Inst. Math. 203, 159-172.

9. A. G. Sergeev and P. Heinzner, The extended matrix disk is a domain of holomorphy, Math. USSR Izvestijav 38 (1992).

10. D. M. Snow, Reductive group actions on Stein spaces, Math. Ann. 259 (1982), 79-97.

11. R. F. Streater and A. S. Wightman, PCT, Spin and statistics, and all that, W. A. Benjamin, Inc., New York-Amsterdam, 1964.

12. V. S. Vladimirov, Analytic functions of several complex variables and quantum field theory, (Russian), Trudy Mat. Inst. Steklov. 135 (1975), 68-80, 256.

13. V. S. Vladimirov, Several complex variables in mathematical physics, Sém. Lelong-Skoda, Lecture Notes in Math. 919 (1982), 358-386.

14. V. S. Vladimirov and V. V. Zharinov, Analytic methods in mathematicial physics, (Russian), Trudy Mat. Inst. Steklov. 175 (1986), 113-133, 247.

15. A. S. Wightman, Quantum field theory and analytic function of several complex variables, J. Indian Math. Soc. (N.S.) 24 (1960/1961), 625-677.

16. B. I. Zav'yalov and V. B.Trushin, On the extended n-point tube, (in Russian), Teoret. Mat. Fiz. 27 (1976), 3-15.

17. X. Y. Zhou, On orbit connectedness, orbit convexity, and envelopes of holomorphy, Izvestija Ran. Ser. Math. 58 (1994), 196-205.

18. $\_$A proof of the extended future tube conjeture (to appear).

Institute of Mathematics, Academia Sinica, Beijing, CHinA

E-mail address: xyzhou@math08.math.ac.cn 